\title{
Daniel Danis en traduction : problèmes de traduction du Chant du Dire-Dire en espagnol chilien ${ }^{1}$
}

\author{
ANDREA PELEGRÍ KRISTIĆ
}

Dans cet article, Pelegrí Kristić propose une étude de la traduction chilienne de la pièce Le chant du Dire-Dire de Daniel Danis, réalisée dans le cadre de la théorie fonctionnaliste du skopos (Vermeer 1978). Cette approche postule que la traduction d'un texte est subordonnée à la fonction (ou skopos) assignée au texte d'arrivée définie suivant le contexte de communication qui sera le sien et suivant l'usage et les usagers auxquels il est destiné. Ce skopos permet ainsi d'établir un projet de traduction concret, qui définit les choix traductifs. Grâce à sa souplesse, nous proposons cette théorie comme une alternative méthodologique efficace pour la traduction de cette pièce, étant donné sa complexité stylistique et esthétique. Particulièrement, notre projet de traduction offre une « troisième voie » entre la littérarité et l'adaptation de la pièce au contexte chilien, en produisant une version chilienne qui essaie de garder les particularités stylistiques et les trouvailles de Danis.

This article offers a study of the Chilean translation of Daniel Danis's play Le Chant du Dire-Dire, which was produced in accordance with the functionalist principles of the skopos theory of translation (Vermeer 1978). This approach subordinates the translation of a text to the function (or the skopos) that is assigned to the target text, defined in conformity with the target text's own communicational context and consistent with its purpose and addressees. This skopos facilitates the establishment of a concrete translation plan that defines the translation choices. For its flexibility, this article proposes this theory as an efficient methodological alternative for the translation of this stylistically and aesthetically complex play. In particular, the translation plan presents a "third way" between a "literary" translation [litterrarité : that is, formal and linguistic properties of a text that distinguish it from other kinds of texts as "literature"] and the play's adaptation to a Chilean context; rather, the translation results in a Chilean version that attempts to retain the stylistic particularities and the expressive inventions found within Danis's play [its littérarité].

La littérature et la question de la langue au Québec ont toujours été inéluctablement liées. Le rapport quelque peu problématique entre les deux infléchit le parcours littéraire de tout écrivain québécois. Gilbert David l'a déjà constaté en parlant plus particulièrement du genre qui nous concerne ici, le théâtre :

[1]e théâtre québécois se retrouve encore et toujours confronté à la question de la langue, lieu chargé de tous les affects [...]. Une espèce de karma linguistique colle à demeure à notre vie théâtrale $[. .$.$] . Le chantier de tout auteur québécois « de souche » [. . . commence et se poursuit dans$ le combat sans fin avec une langue déterritorialisée et en même temps omniprésente (63-64). 
L'œuvre du dramaturge québécois Daniel Danis n'échappe pas à ce constat. Bien que chez lui ce rapport étroit à la langue soit plutôt fécond et positif, éloigné des querelles linguistiques et de cette "surconscience linguistique " poignante dont parle Lise Gauvin (8), la langue demeure toujours au cœur de sa dramaturgie. Elle est non seulement le matériau de création, mais surtout la charpente qui soutient tous les éléments de ses pièces, soit la fable, les personnages, les thématiques et, bien sûr, le style d'écriture. De Cendre de Cailloux à $e$, roman dit, en passant par Le langue-à-langue des chiens de roche, l'univers danisien est traversé par une vision métaphysique et transcendantale de la langue. ${ }^{2}$

Ce n'est cependant que dans la pièce Le chant du Dire-Dire (publiée sous forme de tapuscrit en 1996, à Paris, et puis produite en 1998, à Montréal) que le leitmotiv de la langue devient central. Le langage proposé par Danis, écartelé entre un parler populaire presque prosaïque et des inventions poétiques tout à fait inouies, sert à construire un univers mythique et magique où la parole devient la force créatrice de tout évènement saillant de la pièce. À travers un jouet inventé, le Dire-Dire, frères et sœur Durant (Rock, William, Fred-Gilles et Noéma), incapables de parler, pourront s'exprimer et verbaliser leurs expériences. Pourtant, le lecteur ou le spectateur constate que cet objet, source de bonheur en apparence, est aussi la source des malheurs de la famille Durant, qui se terminent avec la mutilation des frères à la toute fin de la pièce. Il faut d'ailleurs remarquer que la pièce est divisée non pas en scènes ou tableaux, mais en "dires ", ce qui renforce l'importance de la parole créatrice à même la structure dramaturgique.

Ces particularités stylistiques et thématiques sont aussi accompagnées d'un complexe dispositif scénique où récit et dialogue, présent et passé, théâtralité et performativité se côtoient constamment. En outre, la langue n'est jamais employée de façon référentielle, réaliste ou mimétique. Par contre, elle devient " enjeu dramatique " puisque " corps et langue sont un : le "parolique" est le terme attribué par l'auteur à cette poétique du corps parlant qui se fait écriture d'une expérience mi-physiologique, mi-onirique d'une blessure-image imprimée dans le corps »(David 80). Danis plonge ainsi le lecteur/spectateur dans un univers " autre » qui le déstabilise et l'oblige à remettre en question les idées reçues sur le théâtre.

\section{Traduire ou ne pas traduire Danis : telle est la question}

En apparence, la dramaturgie de Danis et la pièce Le chant du Dire-Dire en particulier semblent être fortement ancrées dans le contexte québécois d'où elles émergent. Dans le programme de la première française du Langue-à-langue des chiens de roche, l'auteur propose un texte sur le rapport corps-parole-Québec relevant plus de la création poétique que d'une simple description de sa pièce ou de son processus d'écriture :

Quelle est cette langue que je parle au Québec et diffère-t-elle de celle écrite dans mes pièces de théâtre ; je veux dire tout de suite et cela sans manquer le point d'interrogation, que langue et corps sont uns [...], j'enfonce mon corps-Québec dans le sol, pour prendre racines, m'interroger sur la langue $[\ldots]$ suis-je un glossaire de quelques mots anciens et de mille anglicismes entremêlés en un savoureux et baveux French Kiss [. . .] mon écriture pédestre recherche-telle l'autochtonie de nos ancrages, le théâtre me prend par le ventre, viscéralement (Danis, cité par Hemmerlé I). 
La relation entre cette langue et son terroir devient ainsi évidente. Pour Danis, ce « corpsQuébec " puise les paroles de cette langue dans la terre, une terre où le français archaïque du Grand Siècle se mélange avec les anglicismes dans ce «baveux French Kiss». Certes, les images dégagées du texte se rapprochent du cliché, mais il n'en demeure pas moins que la langue au Québec est à la croisée de toutes ces tensions.

Si ce langage danisien, ce " parolique », est si intimement lié à son propre contexte d'énonciation-le Québec-, il semblerait difficile d'envisager une traduction de cet auteur, en raison de cette spécificité en apparence « locale » et de sa complexité stylistique, notamment dans Le chant du Dire-Dire. Pourtant, cette pièce connaît déjà neuf traductions, dont celle, assez célèbre, de Linda Gaboriau en anglais. La question du " traduisible » devient ainsi accessoire. Malgré la difficulté qu'implique la traduction de cette pièce dans n'importe quelle langue, l'intérêt qu'elle suscite chez les traducteurs et les metteurs en scène partout dans le monde démontre que cette spécificité stylistique de Danis transgresse les frontières linguistiques, politiques ou idéologiques. En effet, chez l'auteur, l'originalité de la parole est bien plus remarquable qu'une filiation réductrice quelconque avec les tensions linguistiques du Québec.

Étant donné les tensions et les problématiques que pourrait susciter une éventuelle traduction du Chant du Dire-Dire, le présent article offre une étude de cas de notre version chilienne de la pièce, réalisée dans le cadre méthodologique d'une théorie fonctionnaliste de la traduction, la théorie du skopos, élaborée par Hans Vermeer en 1978 ( $A$ Skopos Theory 3-20; Skopos and Comission 22I-32) d'après la théorie de l'action traductionnelle de Justa Hölz Mänttäri (1984). ${ }^{3}$

Prenant en considération la complexité stylistique et l'hybridité de la pièce, les approches plus traditionnelles de la traduction théâtrale semblent peu convenir pour affronter sa traduction. Même si, dans les dernières années, les études sur la traduction théâtrale se sont diversifiées et multipliées, en offrant des approches novatrices, ${ }^{4}$ une bonne partie des ouvrages et des essais publiés continue à offrir des stratégies normatives pour la démarche traductrice (Regattin 157; Pelegrí 88). Certaines de ces études vont même proposer des notions pour guider le processus de traduction, telles que jouabilité (ou sa version anglophone, playability), théâtralité, performabilité (ou performability en anglais) ou gestualité. Ces concepts manquent de clarté, et varient selon l'usage ou l'auteur qui les propose. Susan Bassnett déclare ainsi :

If a set of criteria ever could be established to determine the "performability" of a theatre text, then those criteria would constantly vary, from culture to culture, from period to period and from text type to text type [...]. It is also significant that the term "performability" first makes its appearence in the twentieth century and then most frequently in connection with theatre texts that are either naturalist or post-naturalist (IO2-3).

Malgré leur utilité dans certains cas particuliers (des pièces appartenant à une esthétique plus réaliste, par exemple), ces notions ne servent qu'à renforcer un lieu commun, à savoir que le texte de théâtre en traduction doit être " facile à dire ", rythmé et naturel, en négligeant les particularités stylistiques, esthétiques et même culturelles d'une pièce donnée. Patrice Pavis, dans son texte sur la spécificité de la traduction théâtrale, critique ces concepts, et ajoute que, d'un point de vue interculturel, il est dangereux d'imposer des normes présup- 
posées de jouabilité, clarté ou audibilité dans le discours théâtral, étant donné la multiplicité des esthétiques et des cultures (I42).

Le langage de Danis, y compris au niveau théâtral, pose une série de problèmes pour n'importe quel traducteur. Tiraillée entre «l'oralité populaire et la littérarité » selon Gilbert David, la langue est truffée d'expressions populaires, d'images poétiques soutenues, qui empruntent à une grande variété de figures de style et de procédés littéraires, tels que le " couplage» des mots, les néologismes, les allitérations ou les rimes. Elle s'éloigne ainsi d'une vision mimétique ou réaliste du théâtre, d'où la nécessité d'échapper aux notions trop prescriptives de la traduction théâtrale.

En présentant certains extraits de la pièce en version bilingue, et en analysant ensuite leurs principaux problèmes de traduction, cet article vise ultimement à démontrer que la valeur d'une traduction de la pièce ne devrait pas être mesurée à l'aune d'une notion restreinte de fidélité (au sens d'une correspondance littérale) au texte de départ. Pourtant, et contrairement à ce que les critiques de Vermeer en pensent, la théorie du skopos ne néglige pas le texte original. À cet égard, la traductologue Christiane Nord signale que le texte traduit a toujours un rapport à l'original, défini en fonction du but assigné à la traduction. Selon ce but, le traducteur établit les critères pour sélectionner les aspects du texte source qui seront préservés et ceux qui seront adaptés. Il y aurait donc un rapport de " fonctionnalité et loyauté » au texte source:

Within the framework of the concept "functionality plus loyalty" a translator can focus on particular ST aspects [...] and disregard others, if this is required by the TT skopos. However, in such cases he would be obliged, in view of his loyalty towards the ST sender, to specify exactly which ST aspects he has taken into account and which he has neglected (29).

Il s'ensuit que, selon les théories fonctionnalistes (et donc celle du skopos), chaque traduction sera jugée adéquate ou non selon le projet de traduction établi à partir de la fonction assignée au texte d'arrivée, c'est-à-dire son skopos. Lorsque nous parlons de "projet de traduction ", nous renvoyons aux définitions données par Antoine Berman dans son ouvrage Pour une critique des traductions, dans lequel le projet de traduction est vu comme une intention consciente qui soutient toute traduction cohérente et qui, de manière semblable aux théories fonctionnalistes, est défini selon la "position traductive ", le contexte cible, et les contraintes du texte original (76).

Dans le contexte de notre démarche, il sera donc nécessaire de définir et d'expliquer clairement quel est le projet qui guide cette traduction en particulier, ainsi que de décrire sommairement son contexte d'énonciation cible.

\section{La théorie du skopos et le projet de traduction : la version chilienne du Chant du Dire-Dire}

La théorie du skopos postule que la traduction d'un texte est une action humaine et, en tant que telle, nécessairement orientée vers un but. Cette action traductionnelle produit un texte d'arrivée (target text) dont le but ou skopos doit être établi préalablement par le traducteur, au besoin avec le donneur d'ouvrage (initiator ou commissioner). Dans certains cas, il est possible 
que le traducteur soit aussi l'initiateur du projet. C'est le cas d'un metteur en scène qui produit sa propre traduction.

Tous les choix de traduction sont subordonnés à ce skopos, c'est-à-dire à la fonction assignée au texte d'arrivée définie suivant le contexte de communication qui sera le sien et suivant l'usage et les usagers (addressees) auxquels il est destiné. Pour Vermeer, tout acte de traduction (translational act) repose sur un skopos. Un même texte de départ peut donc aboutir à des traductions différentes si elles répondent à des usages différents. Autrement dit, le texte original n'est plus l'unique repère du traducteur : selon cette approche, on tient compte du fait que le texte traduit n'a pas forcément la même finalité que l'original. Par conséquent, chaque projet de traduction exige une analyse préalable de la fonction du texte d'arrivée et du contexte de communication qui lui est propre : « [w] hat the skopos states is that one must translate, consciously and consistently, in accordance with some principle respecting the target text. The theory does not state what the principle is : this must be decided separately in each specific case [...]"(Vermeer, Skopos and Comission 228).

Comme cette théorie suppose que le texte de départ n'a plus un statut sacré pour le traducteur, la notion de "fidélité » est remplacée par celle d' " adéquation » au skopos du texte cible. D'après certains critiques de Vermeer, cette théorie préconiserait une adaptation systématique du texte source. Pourtant, cette méthodologie de traduction n'exclut pas une version en conformité exacte avec l'original. Autrement dit, la littéralité ou l'adaptation ne sont que des modalités possibles sur le spectre de toutes celles qui existent.

En appliquant les principes généraux de la théorie du skopos à un projet de traduction théâtrale, il s'avère fondamental de prendre en considération certains facteurs inexistants dans d'autres types de texte, dont le projet de mise en scène et la compagnie ou le metteur en scène chargés de monter la pièce. Notre skopos pour la version chilienne du Chant du DireDire envisage une future mise en scène de la pièce en 2014 à Santiago du Chili assurée par une compagnie locale émergente, Tiatro, dirigée par les comédiens Mauricio Quevedo et Andrea Pelegrí Kristić et créée en 2006.

La mise en scène envisagée pour ce projet cherchera principalement à mettre en valeur la recherche langagière propre à l'esthétique danisienne et à y subordonner tous les autres éléments constitutifs de la fable. Le projet scénique mettra en relief les multiples fonctions de la parole dans la pièce, du triple point de vue thématique et symbolique aussi bien que stylistique. Ainsi, notre projet de traduction a pour but principal de trouver la logique interne des différentes figures de style et inventions langagières de Danis, en profitant de la liberté stylistique de l'auteur, pour les reproduire dans la version chilienne. Il ne s'agit pas pourtant de trouver des équivalences dans la traduction, car, comme le signale Mary-Snell Hornby, cette notion est contestable:

[Ce concept] est essentiellement abstrait, statique et unidimensionnel; il passe à côté de la dynamique oscillante de la langue et reste illusoire. Sa validité se limite à quelques domaines de la traduction technique, qui repose sur une identité conceptuelle indépendante du contexte (cit. dans Pavis I65).

De ce fait, une des consignes du projet de mise en scène et, en conséquence, de la traduction, est de ne pas situer l'action de la pièce dans un contexte spécifiquement chilien et, sur 
le plan linguistique, de ne pas rendre le vernaculaire québécois-cette oralité dont parle David-par des «équivalences » chiliennes, justement pour éviter de transformer la pièce en portrait réaliste d'une classe sociale donnée : en général, l'emploi du vernaculaire dans la dramaturgie chilienne incite à une lecture politico-sociale, difficile à évacuer. L'emploi de vernaculaires chiliens dans le théâtre est devenu une pratique courante chez l'auteur Juan Radrigán. Dans ses pièces, les différents vernaculaires de Santiago ou de certaines régions rurales ont un statut plus ou moins semblable à celui du joual dans la dramaturgie québécoise des années i960 et 1970.5 Pourtant, le rapport entre langue populaire, langue vernaculaire, pauvreté et marginalité est très prononcé chez Radrigán ; ses personnages et leur langage font clairement référence à un groupe social typiquement chilien (Hurtado et Piña 2I). Ce rapport quelque peu indissociable entre langue vernaculaire et classe sociale est aussi repris par un autre dramaturge plus jeune, Luis Barrales. En 2007, sa pièce H.P (Hans Pozo) raconte l'histoire d'un garçon de banlieue tué et mutilé par son amant, un homosexuel refoulé. Les différents monologues constituant la pièce utilisent un lexique et une syntaxe qui reproduit un type de vernaculaire associé aux personnages et à leur classe sociale. Il est donc très difficile de traduire en vernaculaire chilien sans que le registre langagier ne ramène constamment le spectateur à une réalité marginale typiquement locale.

Cette décision de ne pas trouver un vernaculaire chilien équivalant dans notre projet de traduction s'avère à notre avis logique si l'on considère que Danis opte sciemment pour situer sa pièce dans un espace-temps indéterminé. Certes, les personnages parlent un français assez québécois, situé probablement à l'époque actuelle, puisque les frères Durant regardent la télé, par exemple. Cependant, la pièce propose une temporalité anhistorique qu'on devrait préserver : « je suis violenté quand on ramène mes personnages dans un endroit historisant. Dans Le chant du Dire-Dire c'est la même chose. Si on historise les personnages, on ne raconte plus leur endroit mythologisé » (Danis, Fabrique 4).

En suivant cette logique, dans notre lecture de la fable de Danis, Le chant du Dire-Dire est l'histoire du retour aux origines de la race humaine, du retour à son état primitif avant la culture, à « un âge d'or »(Blonde IO3). Cette histoire est présentée métaphoriquement à travers celle des frères Durant. À la fin de la pièce, après une série d'aventures, de désastres et d'accidents, les frères Durant fuient le monde civilisé, s'enfoncent dans la forêt pour se percer les tympans, se crever les yeux et se couper la langue. Ils atteignent alors un état de neutralité, de pureté, tel que Danis l'annonce en exergue : "Loin de tout regard, de tout bruit, de toute parole prononcée, [. . . l l'être peut secrètement s'unir à son âme-étant pour atteindre une indifférence à la vie et à la mort afin de laisser monter, en son corps, la pureté» (Le chant, 9 ; nous soulignons). Cette mutilation, qui met fin non seulement à la fiction recréée par les personnages, mais aussi à leur vie dans la fable, symbolise la conclusion d'une quête humaine vers la pureté.

C'est cette quête de pureté, de neutralité et d'austérité que nous voudrions mettre en valeur dans la mise en scène. La parole tiendra donc le rôle principal, en construisant l'espace, le temps et les personnages à travers les rythmes, la musicalité des mots et les figures de style proposés par Danis. Ainsi, la conception du décor, de l'espace et des costumes cherche avant tout à ne pas situer les personnages dans un endroit, pays ou société quelconques, comme nous l'avons indiqué précédemment. De plus, l'utilisation d'accessoires sera limitée, puisque la parole construit les espaces dramatiques à travers des figures de style 
telles que l'hypotypose ou la personnification. La narration suffit pour créer cet univers mythique, l'inclusion d'éléments référentiels dans le décor et les costumes ne ferait qu'illustrer et surcontextualiser la pièce.

Notre projet de traduction pose cependant quelques problèmes auxquels il faut s'attaquer : comment est-il possible de produire une version pour un contexte spécifique (une mise en scène de la pièce pour un certain public à Santiago du Chili en 20I4) sans situer nécessairement le texte de Danis dans un contexte " chilien ", autrement dit, sans évacuer son étrangeté et sa propre spécificité culturelle, mais sans essayer en même temps de faire une version dans un espagnol normatif, voire " neutre »? Car, malgré ce que certains donneurs d'ouvrage ou metteurs en scène pensent lorsqu'ils commandent une traduction, ${ }^{6}$ il n'existe pas de registre de langue neutre : lorsque la pièce est traduite et montée dans un contexte quelconque, elle est déjà naturalisée, domestiquée et adoptée par la culture cible (Aaltonen 29 ; Venuti 67-68). Pour tenter de résoudre ce paradoxe, nous proposons une «troisième voie " pour rendre le vernaculaire québécois en espagnol : l'utilisation de néologismes « à la Danis » et l'emploi sélectif de certaines tournures chiliennes (autant au niveau lexical que syntactique) pour éviter une illusion de neutralité ou de traduction espagnole importée.

Pour appliquer la première stratégie, nous nous appuyons sur la logique du texte danisien. Lauteur façonne et retravaille la langue, le traducteur pourrait-il lui aussi employer ce même procédé appliqué aux langages vernaculaires? Suivant la logique du texte et dans une vue d'ensemble, ces inventions seraient cohérentes dans les macrostructures du texte cible. Ce procédé semble être approprié au skopos établi préalablement. Dans le cas de notre deuxième stratégie, quoique la pièce ne soit pas adaptée pour être localisée dans une région chilienne quelconque, nous cherchons à éviter l'utilisation d'un lexique étrange ou peu courant, sans pour autant employer des tournures trop locales.

\section{El canto del Dile-Dile : des exemples de traduction}

Les exemples de traduction cités par la suite sont tirés du deuxième « dire » de la pièce, intitulé «Les traces du chaos dans la maison Durant. »Cet extrait a été spécifiquement choisi car il présente de nombreux éléments caractéristiques de la pièce : les espaces de narration, la coexistence des registres dramatique et narratif, plusieurs figures de style, entre autres. Avant d'entreprendre la traduction, et une fois que le skopos et le projet de traduction ont été établis, nous avons entamé une étude textuelle approfondie, en suivant des modèles d'analyse textuelle propres aux théories de traduction fonctionnalistes, notamment celui de Christiane Nord (70-75). Faute d'espace, nous reproduirons seulement les analyses relatives aux exemples choisis. Bien que les exemples qui suivent sont tirés exclusivement du " dire » indiqué précédemment, certains choix de traduction généraux doivent être présentés avant d'examiner ce « dire » en particulier.

En premier lieu, les prénoms des personnages ont été conservés tels quels (dans le cas de Noéma et Rock) ou adaptés (Fred pour Fred-Gilles et Willy pour William). La traduction des noms propres présente toujours des problèmes pour la traduction, car, stricto sensu, il n'y a pas d'équivalence dans une autre langue et ce serait donc un cas de figure intraduisible, tel que l'a déjà remarqué Derrida au sujet de la traduction de noms tels que «Dieu » ou «Babel » (224-25). En outre, la traduction ne cherche pas à situer l'action au Chili et veut éviter au 
public local un effet de mise à distance. Les noms Fred-Gilles et William ont été adaptés pour éviter aux comédiens des syllabes compliquées à prononcer (le son en français de la syllabe « gi » n'existe pas en espagnol).

Pourtant,-et voici une première contradiction-le nom Durant a été traduit par Durante en espagnol, puisque l'auteur fait un jeu de mots dès le début de la pièce déterminant le destin quelque peu tragicomique des Durant : "C'est à partir de ce moment qu'on a eu le surnom des Durant l'orage » (I9). On a donc choisi de garder cet effet comique au lieu de reproduire le nom français.

Suivant la lecture du « dire », il est possible de repérer la quantité de figures de style utilisées par l'auteur, ce qui représente un défi de taille pour le traducteur. Dans ce dire seulement, Danis utilise les figures suivantes : allitérations, assonances, homéotéleutes, personnifications et hypotyposes, sans oublier les métaplasmes qui servent à reproduire des marques d'oralité (la contraction « t'as » au lieu de « tu as ", ou «C'ui-là » au lieu de « celui-là »). Le phénomène le plus intéressant relève de la création des mots. Danis utilise, surtout dans ce deuxième "dire » des doublons des mots ou « couplages ", d'après Marion Chénetier-Alev (243), pour créer de nouveaux lexèmes : "pensé-rêvé ", "soudés-collés ". Ces couplages ont plusieurs fonctions : Gilbert David note par exemple que parfois ce procédé est employé comme une " autocorrection lexicale " (79). Il sert aussi à regrouper dans un seul souffle deux idées ou plus, pour ainsi mettre au même niveau d'importance une série de mots qui, dans d'autres circonstances, seraient dits séparément. Pour le comédien, le couplage offre plusieurs possibilités rythmiques et vocales. Pour le traducteur, un défi s'impose : suivant le projet de traduction, comment peut-en garder la rythmique sans pour autant sacrifier le sens? Considérant que l'auteur crée en général des couplages de deux mots, en voici un exemple tout à fait radical, où il combine huit mots :

LES TROIS. Comme une flèche ramasseuse, l'éclair pointu-tordu a enfilé les deux cœurs pêle-mêle-rouge-sang-père-mère-bleu-flash. (I6)

Dans ce texte, dit en chœur, l'auteur regroupe une série d'images dans un seul lexème où chaque mot n'a plus de signification sans celui qui l'accompagne. Ce collage crée une image, comme s'il s'agissait d'un tableau de la mort des parents. Il a aussi une rythmique particulière, car certaines combinaisons forment des homéotéleutes (pêle-mêle, père-mère), alors que d'autres expressions relèvent du phénomène des onomatopées (flash). En outre, les huit mots qui composent le " grand mot " sont d'une ou deux syllabes, créant ainsi une rythmique accentuelle assez régulière : l'accent tombe toujours en première syllabe. Dans la traduction, le nombre de mots a été préservé, en introduisant deux mots pour pêle-mêle, «ruido» (bruit) et «caos» (chaos):

LOS TRES. Como una flecha recogedora, el rayo puntudo-torcido ensartó los dos corazones ruido-caos-rojo-sangre-padre-madre-azul-flash.

La rythmique du texte original a ainsi été gardée. Faute de préserver l'homéotéleute de "pêle-mêle » et " père-mère ", une autre homéotéleute a été proposée en espagnol : «sangre, padre, madre ». Évidemment, pour les autres couplages doubles, le même critère s'est imposé 
pour leur traduction : garder la quantité des syllabes si possible, ainsi que préserver les homéotéleutes ou les allitérations.

Une autre invention lexicale proche des couplages, moins présente dans cette pièce mais importante dans l'ensemble de sa dramaturgie, s'avère les néologismes créés par Danis. Pour Gilbert David, ces néologismes sont l'exemple manifeste de cette double filiation avec l'oralité populaire, la littérarité et les inventions poétiques (79). D'une certaine façon, cette inventivité insuffle au langage littéraire un effet d'oralité ludique, en accord avec l'attitude un peu enfantine des personnages, mais aussi de la pièce. Un exemple qui apparait dans ce deuxième dire est le mot «municipiens », rendu en espagnol par «municipiano ». Le choix de traduction dans ce cas a été de suivre la structure du néologisme danisien, lequel a été probablement formé par l'union du mot " municipalité » et le suffixe "-ien ", qui sert à indiquer l'appartenance à un certain lieu. En général, cette logique a été respectée pour tout autre néologisme : on a essayé de décortiquer le sens de chaque mot pour reproduire cette même logique en espagnol.

Loralité populaire n'est pas seulement présente à travers les néologismes mais aussi et surtout par l'emploi de certains québécismes, comme « garrocher». Certes, l'emploi du vernaculaire québécois est moins présent dans cette pièce et dans ce "dire " en particulier, mais il demeure très présent, surtout dans les fragments dialogués. Suivant notre projet de traduction et le skopos préétabli, pour "garrocher ", par exemple, le mot «tirar » a été choisi. Ce mot est très courant en espagnol, peu importe la région, mais il a été retenu au lieu de son synonyme, trop soutenu, «lanzar».

Voici un autre exemple d'une expression typiquement québécoise ainsi que notre traduction proposée, où nous avons combiné l'utilisation d'une expression plutôt chilienne avec un lexique moins situé géographiquement :

Et les tonnerres s'en allaient, contents de leur monstruosité, se claquant les bretelles de leur hauteur de nuages devant des éclairs, encore tous excités, qui piquaient des clins d'œil de clownerie (I7).

En décortiquant ce fragment, on peut noter que l'auteur fait ici allusion à l'expression québécoise "se péter les bretelles ", qui signifie "se réjouir (de son succès), se complaire " (DesRuisseaux 78). Le verbe "claquer " a été traduit par "chasquear»; pour rendre l'expression "se péter les bretelles", une locution chilienne (la seule dans la traduction) a été introduite : "creerse la muerte ». Bien que ce projet de traduction n'ait pas pour objectif de situer la pièce au Chili, cette expression est transparente ; sa connotation chilienne n'est pas évidente, si l'on pense à d'autres locutions semblables, comme "creerse el boyo del queque " (" se croire le trou du cul») ou "quebrarse » (littéralement, « se briser »).

En gardant le geste de faire claquer les bretelles, l'image suivante des clowneries, traduite cette fois-ci par un verbe, "payasear », est complétée par l'image des bretelles. Létrangeté de ce fragment est évidente, mais l'auteur déstabilise constamment le spectateur/lecteur avec des images bizarres, contradictoires. La traduction cherche à préserver cette étrangeté mais sans trop mettre à distance le spectateur chilien.

Suivant cette même logique, nous avons toujours privilégié l'emploi d'un lexique moins soutenu et plus proche du public chilien. Ainsi, des mots tels que " argent » ou " plombier» 
ont été traduits par " plata " et « gasfiter » respectivement, au lieu de leur traduction plus universelle, " dinero » et « plomero ». Dans le premier cas, "plata » est assez répandu dans tout l'Amérique Latine, tandis que «gasfiter " (probablement, un néologisme du concept anglais " gas fitter ») est plutôt chilien. Ces deux exemples démontrent comment notre projet cherche un espagnol chilien, mais pas spécifique à une classe sociale, justement pour éviter les connotations sociopolitiques décrites précédemment.

\section{Et au futur, le dernier pas du chemin : la traduction mise à l'épreuve}

Tout au long de cet article, nous avons essayé de prouver l'efficacité et l'utilité de la théorie du skopos (et des approches fonctionnalistes en général) pour établir des projets de traduction dans le domaine de la dramaturgie. Vu que les approches plus traditionnelles sur la traduction théâtrale s'avèrent trop prescriptives pour des textes moins naturalistes, la théorie du skopos offre un cadre bien plus souple pour notre démarche traductive. Ceci dit, cette recherche n'est pas réalisée dans le vide et se situe dans la lignée de travaux sur la traduction théâtrale des dernières années ; autant dans le domaine théorique que pratique en traduction, les contributions d'Annie Brisset (1990), Glen Nichols (2002) ou Louise Ladouceur (1995), pour ne nommer que quelques-uns, proposent de nouvelles approches et perspectives sur le sujet; il faudrait mentionner aussi, dans le contexte européen, Sirkku Aaltonen (2000). Ces auteurs ont élargi le champ de recherche en se concentrant sur les normes traductives, les contraintes sociales, politiques et culturelles, et sur la position occupée par la traduction dans les systèmes littéraire et théâtral de leurs pays. Ainsi, les travaux de Brisset et Ladouceur, tributaires des théories fonctionnalistes de traduction ainsi que de la théorie des polysystèmes de l'École de Tel-Aviv, ont largement influencé notre démarche.

Dans la sphère pratique, la traduction anglaise de la pièce signée par Linda Gaboriau (Song of the Say-Sayer) ainsi que la version mexicaine de Boris Schoemann (El canto del DimeDime) nous ont offert des pistes possibles pour notre propre traduction, particulièrement celle de Gaboriau. Les choix traductifs de Song of the Say-Sayer révèlent une connaissance approfondie de la dramaturgie danisienne et du rapport corps-parole dans ses pièces : Gaboriau ajoute même une didascalie au début de la pièce pour guider le travail de comédiens dans un passage donné (Gaboriau cit. par Whitfield 297). La lecture de cette version nous a fourni l'idée d'une possible «troisième voie » pour notre propre traduction.

Bien qu'à ce stade de la recherche, on puisse évaluer les choix de traduction selon le skopos établi, une autre dimension s'avère impossible à apprécier pour l'instant : la mise en scène du texte et sa réception. La traduction d'une pièce de théâtre ne se termine pas au moment d'imprimer l'ébauche finale, surtout si cette traduction est effectuée en fonction d'une mise en scène. Selon le schéma de Pavis (137-39), le texte devrait continuer son cheminement à travers les étapes $\mathrm{T}_{3}$ et $\mathrm{T}_{4}$, à savoir la concrétisation scénique et la concrétisation réceptive, c'est-à-dire le moment où le texte achève réellement son parcours et parvient jusqu'au public cible. C'est seulement à ce moment-là qu'on pourra comprendre l'impact de la pièce sur le public chilien, son niveau d'acceptation, et aussi de quelle façon les choix de traduction seront appréciés ou non. Est-ce que les néologismes ou l'emploi de certaines tournures chiliennes ou de l'Amérique Latine permettront un rapprochement du public chilien 
avec l'univers de Danis ou, au contraire, produiront-ils une mise à distance? Est-ce qu'une troisième voie est vraiment possible, ou ne s'agit-il que d'une illusion, puisque toute pièce traduite est naturalisée et domestiquée dans son contexte cible? Cela reste à voir.

Afin de répondre à ces questions, les recherches à venir sur le sujet devraient non seulement porter sur l'effet de la traduction en termes linguistiques, mais surtout se concentrer sur le travail des comédiens sur cette parole particulière, sur le travail de mise en scène et sur l'intégration de la dramaturgie danisienne au contexte chilien, habitué à d'autres dramaturgies étrangères et peu familiarisé avec les auteurs canadiens. Le style particulier de Danis oblige le metteur en scène à inscrire le travail des comédiens dans une logique non réaliste, éloignée du mimétisme et d'une identification entre personnage et comédien. L'œuvre d'un metteur en scène comme Robert Wilson montre les différentes possibilités sonores et physiques à explorer par les comédiens. La future mise en scène devra tenir compte de l'esthétique particulière de Danis et devra donc défier certaines tendances plus réalistes d'une partie du théâtre chilien, en rejoignant ainsi les compagnies et les théâtres plus ouverts aux explorations formelles et esthétiques. Il nous semble que dans de pareilles conditions, les choix de traduction proposés pourraient, au moins de façon théorique, aboutir au succès.

\section{Notes}

I Ce texte développe les idées exposées dans la conférence « Défis de traduction pour Le chant du Dire-Dire en espagnol chilien ", présenté au congrès annuel de la SQET, le II mai 20II. Ce travail de recherche a été fait dans le cadre de notre maitrise en théâtre (théorie théâtrale et dramaturgie) réalisée à l'Université d'Ottawa. L'article est largement inspiré de notre mémoire, déposé en 20 II.

2 Actuellement, Daniel Danis entame une nouvelle recherche dramaturgique qui donne aux nouvelles technologies une place prépondérante. Lors d'une table ronde sur la création artistique au Festival TransAmériques de 20II, Danis a expliqué que l'omniprésence de la virtualité dans la culture l'obligeait à envisager la création artistique d'une autre manière, d'où la transformation de son écriture : lacunaire, trouée, celle-ci est complétée par des projections et des images. En ce sens, le constat de cette importance prépondérante de la langue chez Danis concerne, au moins de manière évidente, ses pièces des années 1990 et du début des années 2000 . Ceci dit, même dans un spectacle comme Mille anonymes, où la parole n'est qu'un des éléments de la mise en scène, l'écriture acquiert aussi un statut quasi mythique ; les dialogues n'illustrent pas une réalité quelconque, mais invitent plutôt le spectateur à creuser, à travers la parole, dans l'imaginaire individuel et collectif. Ce ne sont bien sûr que des hypothèses sur cette nouvelle "étape » de la dramaturgie danisienne. Nous devrons l'exclure de cet article car elle échappe à son sujet central.

3 Pour plus d'informations sur cette théorie et ses rapports avec les théories fonctionnalistes, voir Translatorisches Handeln de la même auteure.

4 En effet, notre démarche méthodologique est tributaire d'autres recherches traductologiques au Canada, au Québec et en Europe, comme nous le verrons au cours de l'article. Pourtant, il est étonnant que, dans le domaine du théâtre, certains auteurs continuent à ressasser les mêmes constats totalisants sur l'oralité du texte dramatique ou la facilité de se le mettre en bouche au moment de déterminer la spécificité de la traduction théâtrale. 
5 Il serait réducteur pourtant de comparer sans nuances le joual et les vernaculaires chiliens, d'abord parce que la situation de l'espagnol chilien est complètement différente de celle du français québécois ; l'espagnol n'est pas dans une situation de précarité géopolitique et culturelle par rapport à une autre langue. Ceci dit, l'utilisation du vernaculaire chez Radrigán n'est pas seulement une manière de situer les personnages dans un contexte, mais plutôt de les légitimer sur le plateau. Il s'agit d'une revendication symbolique, où la langue devient aussi poésie. Et dans ce sens, le joual de Tremblay (d'après les propos de Lise Gauvin, 2008) ressemble au vernaculaire de Radrigán.

6 En tant que traductrice au Chili, nous avons reçu maintes fois des commandes de traduction vers un espagnol «neutre ", évitant les vernaculaires, les sacres ou tournures de langue typiquement chiliens, sans avoir pour autant d'autres instructions plus précises par rapport à cette prétendue neutralité. La tâche du traducteur dans ce contexte s'avère extrêmement difficile.

\section{Ouvrages cités}

Aaltonen, Sirkku. Time-sharing on stage. Drama, Translation in Theatre and Society. Sidney : Multilingual Matters, 200o. Imprimé.

Bassnett, Susan. «Translating for the Theatre : the Case Against Performability. "TTR : Traduction, terminologie, rédaction 4.I (1991): 99-III. Imprimé.

Berman, Antoine. Pour une critique des traductions : Fohn Donne. Paris : Gallimard, 1995. Imprimé.

Blonde, David. «De Gauvreau à Danis : repères pour une poétique de la langue théâtrales ", mémoire de maitrise, Université d'Ottawa, Département de lettres françaises, 2003. Imprimé.

Brisset, Annie. Sociocritique de la traduction. Théâtre et alterité au 2uébec (1968-1988). Longueuil : Le Préambule, 1990. Imprimé.

Chénetier-Alev, Marion. Loralité dans le théâtre contemporain : Herbert Achternbusch, Pierre Guyotat, Valère Novarina, Fon Fosse, Daniel Danis, Sarah Kane. Lille : ANRT, 2006. Imprimé.

Danis, Daniel. «La Fabrique du dialogue I : Daniel Danis. » Revue électronique du théâtre de la Colline 2 (2005) : s.p. Web. 2 août 2010.

—. Le chant du Dire-Dire. Montréal : Leméac, 2006. Imprimé.

David, Gilbert. « Le langue-à-langue de Daniel Danis : une parole au corps à corps. » Études françaises 43.I (2007) : 63-8I. Imprimé

Derrida, Jacques. «From Des Tours de Babel. » Theories of translation. An anthology of essays from Dryden to Derrida. Dir. Rainer Schulte et John Biguenet. Chicago : U de Chicago P, 1992. 218-27. Imprimé.

DesRuisseaux, Pierre. Dictionnaire des expressions québécoises, Montréal : Bibliothèque Québécoise, 2009. Imprimé.

Gauvin, Lise. Langagement. Lécrivain et la langue au 2uébec. Montréal : Boréal, 200o. Imprimé.

Hemmerlé, Marie-Aude, « La parole-matière : le statut de la parole dans trois pièces de Daniel Danis. Cendre de cailloux, le Langue-à-langue des chiens de roche et Le chant du Dire-Dire ", mémoire de maîtrise, Département des études théâtrales, U de la Sorbonne Nouvelle-Paris III, 200I-2002. Imprimé.

Hurtado, María de la Luz et Juan Andrés Piña. Préface. Hechos consumados. Teatro i o obras. De Juan Radrigán. Santiago : LOM, 1998. Imprimé.

Ladouceur, Louise. «Normes, fonctions et traduction théâtrale. » Meta : Fournal des traducteurs/Meta : Translator's fournal 40.I (1995) : 3I-38. Imprimé. 
Holz-Mänttäri, Justa. Translatorisches Handeln. Theorie und Methode. Annales Academiae Scientarum Fennicae. Ser. B 226. Helsinki : Suomalainen Tiedeakatemia, 1984.

Nichols, Glen. «Trading Partners : New Views on Theatre Translation in Canada. » TTR: Traduction, terminologie, rédaction I5.I (2002) : II7-35. Imprimé.

Nord, Christiane. Text Analysis in Translation Theory. Methodology and Didactic Application of a Model for Translation-Oriented Text Analysis. Amsterdam : Rodopi, I991. Imprimé.

Pavis, Patrice. "Vers une spécificité de la traduction théâtrale : la traduction intergestuelle et interculturelle ». Le théâtre au croisement de cultures. Paris : JosCorti, 1990. 135-70. Imprimé.

Pelegrí K., Andrea. «La especificidad de la traducción teatral : ¿mito o realidad? »Apuntes 137 (2012): 87-Ior. Imprimé.

Regattin, Fabio. "Théâtre et traduction : un aperçu du débat théorique. " L'Annuaire théâtral 36 (2004) : 156-71. Imprimé.

Venuti, Lawrence. The Scandals of Translation. Towards an Ethics of Difference. London : Routledge, I998. Imprimé.

Vermeer, Hans. A Skopos Theory of Translation (Some Arguments for and Against). Heidelberg: TEXTconTEXT, 1996. Imprimé.

—. «Skopos and Comission in Translational Action». Translation Studies Reader. Dir. Lawrence Venuti. London : Routledge, 2000. 221-32. Imprimé.

Whitfield, Agnès, dir. Writing Between the Lines. Portraits of Canadian Anglophone Translators. Waterloo : Wilfrid Laurier UP, 2006. Imprimé. 